\title{
Real time simulation and performance measurement of hydro-turbine governing systems
}

\author{
Simulation en temps réel et mesure des performances des systèmes de com- \\ mande des turbines hydrauliques
}

\author{
par Zhaohui Li, Zelong Wang \\ Dept. of Electric Power Engineering - Huazhong Univ. of Science \& Technology (China) \\ B. Maschke \\ Laboratoire d'Automatisme Industriel - Conservatoire National des Arts et Métiers
}

La simulation joue un rôle important dans le développement des technologies et des réalisations industrielles d'aujourd'hui. Les techniques de simulation en temps réel et de mesure des performances des systèmes de commande des turbines hydrauliques est étudié depuis quatre ans. Un système a été développé et appliqué avec succès à la conception, à la fabrication et l'exploitation de commandes de turbines hydrauliques.

Les développements futurs de ces techniques de simulation en temps réel et leur application dans la conduite, la maintenance et la gestion techniques de centrales hydroélectriques sont également développés.

\section{I — INTRODUCTION}

A successful industrial control system usually needed to be designed and modified over and over. Prototypes had to be manufactured and tested in field. This used to be an expensive long procedure.

Simulation techniques, especially digital simulation techniques, are developing very fast in recent years, along with the rapid evolution on computer science and engineering. According to time characteristics, simulations can be categorized into real time simulation and different time simulation.

In the domain of automatic control, different time simulation is mainly employed at the design stage for control strategy study. Many software packages of different time simulation for control system design are available on market. More detailed (usually more precise) models can be used for this kind of simulation.

Real time simulation must run in the same rhythm as the actual simulated system. So the simulation time becomes very crucial. The precision of the models is usually limited by the computing power.

Real time simulation can be comprehensively applied to : - the design stage for either the control strategy study or the verification of the control strategy implementation in prototype. This application will largely shorten the design period and reduce the design cost.

- the manufacturing stage for quality control. The control- ler can be tested and the performance of control system can be measured in manufacturing workshops.

- and the operation stage for training the operators, for the maintenance of equipment and for technical management.

So it's fair to say that simulation techniques have accelerated the development of modern technologies and industries.

A hydro turbine governing system is composed of two major parts : governor (controller + actuator) and hydro electric generating unit as presented in Figure.1.

Here, $f_{r}$ stands for the speed set point, $p_{r}$ for the power set point, $y_{r}$ for the wicket gate opening set point, $y$ for the wicket gate opening, $h$ for the water pressure, $q$ for the water flow, $f$ for the speed of turbine-generator group, $p$ for the power output of generator.

The performance and reliability of governor directly determines the safety and the stability of hydro-electric generating unit, and even have influence on that of electric grid. Furthermore the characteristics of hydro-electric generating units are very different each other. So each governor, as well as its controller, should be comprehensively tested and the performance should be measured before and during its service on a specified hydro-electric generating unit. A Hydro Turbine Governing system real time Simulation and performance Measurement System (HTGSMS) was developed in this motivation. 


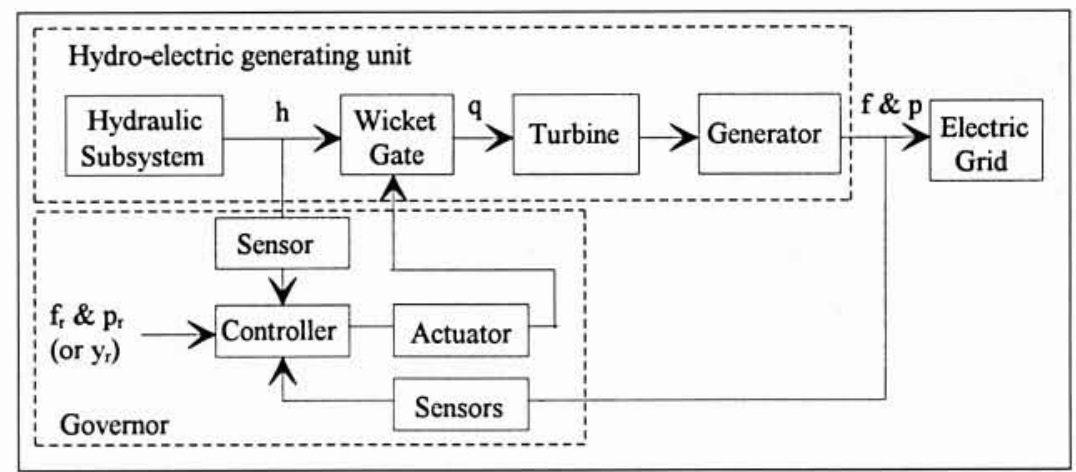

1. Hydro Turbine Governing System.

Here SW1 and SW2 are software switches controlled by operator accessable flags and SW3 is directly operated by man force. Five possible configurations, as listed in Tab.1, can be selected via these three switches.

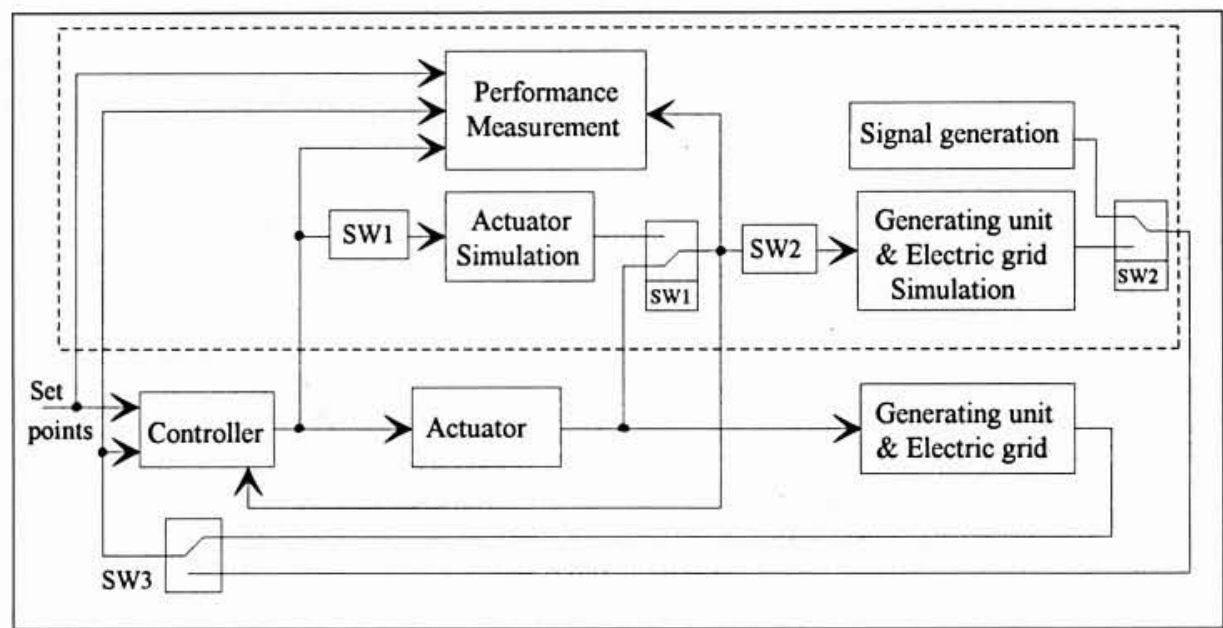

2. HTGSMS.

\begin{tabular}{|c|c|c|l|}
\hline SW1 & SW2 & SW3 & \multicolumn{1}{c|}{ Configuration } \\
\hline 0 & 0 & 0 & Controller+Actuator for static tests \\
\hline 0 & 0 & 1 & Controller+Actuator+Unit for dynamic tests (as shown in Fig. 2) \\
\hline 1 & 0 & 0 & Controller+simulated Actuator for static tests \\
\hline 0 & 1 & 0 & Controller+Actuator+simulated Unit for dynamic tests \\
\hline 1 & 1 & 0 & Controller+simulated Actuator+simulated Unit for dynamic tests \\
\hline
\end{tabular}

Table 1. The possible configurations of the HTGSMS.

The real time simulation scheme of the hydro-turbine governing system is described in section 2 . The performance requirements on hydro-turbine governors are presented and measurement methods of these performances are studied in section 3 . The experiment results are demonstrated in section 4. The further development of these techniques and their application to the operation, maintenance, technical management of hydro-power plants are discussed in section 5 .

\section{II $\square$ REAL TIME SIMULATION}

The controller is assumed to be always available. Only the actuator and the hydro-electric generating unit are needed to be simulated.

\subsection{Real time simulation of the actuator}

The actuator mainly consists of an electric-hydraulic converter, a hydraulic amplifier, two mechanic arms, and a sensor for the feed-back of the wicket gate opening. It is a servo-mechanism with non-linear properties such as deadzone, saturation and backlash.

Each component is independently modeled. All "components" are linked together according to the sequence of signal flow, by interfacing the output of previous component to the input of next component as shown in Fig.3.

The input of the "actuator" is interfaced to the wicket gate opening control output of the controller and its output is connected to the "hydro electric generating unit".

A sampling period $T$ (for example 4 milli-seconds) is selected and it is synchronized with standard clock during the simulation.

The characteristic parameters including the dead zone $d_{v}$ the saturation $l m t$, the backlash $b l$, the time constant $T_{y}, T_{s}$, and the gain of the amplifier $K_{y}$ can be tuned for each particular application. Imt may be the function of the opening $y$ 


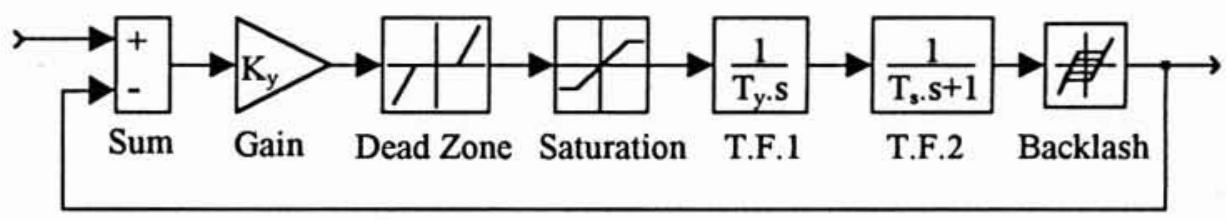

3. Actuator of Hydro Turbine Governor.

and operating direction of the wicket gate in order to set different operation speed limits of mechanic arms.

\subsection{Real time simulation of the hydro-electric genera- ting unit}

The hydro electric generating unit fundamentally consists of hydraulic subsystems (pipelines or tunnels, penstock, and also surge tank(s) in some cases), turbine and generator (and electric grid when the generator is synchronized).

The hydraulic subsystem, turbine, and generator are independently modeled. They are linked together by considering the signal exchanges among them. The input of the "unit" is connected to the output of actuator. Its outputs and some states are fed back to the controller.

\subsubsection{Hydraulic Subsystem}

The hydraulic subsystem is the most complicated part to model and furthermore it is different for each generating unit. Here gives a modeling example of simplest case as shown in Fig.4.

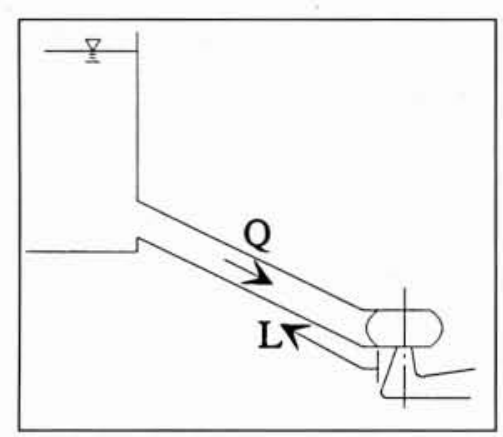

\section{Hydraulic Subsystem of a Hydro-electric Generating Unit.}

Assume : the length of pipeline is counted in direction from penstock to reservoir ; the reservoir is infinitely large. The dynamics of the water flow $Q(L, t)$ and the pressure $H(L$, $t$ ) at Section $\mathrm{L}$ of pipeline, which is situated $L$ far from penstock, can be described by following equations :

$$
\begin{aligned}
& T_{w} \frac{\partial q(l, t)}{\partial t}=\frac{\partial h(l, t)}{\partial l}-f_{l}\left(q_{0}^{2}+2 q_{0} q(l, t)\right) \\
& \frac{4 T_{w}}{T_{r}^{2}} \frac{\partial q(l, t)}{\partial l}=\frac{\partial h(l, t)}{\partial t} \\
& q(l, 0)=0 \\
& h(l, 0)=-f_{l} q_{0}^{2}(1-l) \\
& h(l, t)=0
\end{aligned}
$$

Here,

$q(l, t)=(Q(L, t)-Q(L, 0)) / Q_{r}$

$h(l, t)=(H(L, t)-H(L, 0)) / H_{r}$

$l=L / L_{r}$

$q_{0}=Q_{0} / Q_{r}$

$$
\begin{aligned}
& T_{w}=L_{r} Q_{r} /\left(g H_{r} F\right) \\
& T_{r}=2 L_{r} / a \\
& f_{l}=\alpha Q_{r}^{2} L_{r} /\left(2 g H_{r} F^{2}\right)
\end{aligned}
$$

$L_{r}$ is total length of the pipeline from penstock to reservoir ; $g$ is the gravity; $a$ is the velocity of the water wave; $\alpha$ is the wall friction coefficient of pipe-line (or tunnel); $F$ is the section surface of pipe-line (or tunnel); $H_{r}$ is the design water pressure ; $Q_{r}$ is the rated water flow; $Q_{0}$ is the initial steady flow at selecting operating point.

The flow and the water pressure in penstock are :

$$
\begin{aligned}
& q=q(0, t) \\
& h=h(0, t)
\end{aligned}
$$

\subsubsection{Turbine}

The turbine is a non-linear component. It can be described by next equation :

$$
\begin{aligned}
& M_{T}=M_{T}(H, n, Y) \\
& Q=Q(H, n, Y)
\end{aligned}
$$

Here $M_{T}, H, n, Y$ and $Q$ respectively represent the mechanic torque output of turbine, water pressure, rotation speed of turbine, wicket gate opening and water flow. Let :

$$
v=\left(V-V_{0}\right) / V_{r}
$$

$V_{r}$ is rated value of $V$ and $V_{0}$ is the value of $V$ at a fixed operating point. $\mathrm{V}$ stands for any of the variables $M_{T}, H, n$, $Y$ and $Q$ and $v$ correspondingly represents $m_{t}, h, f, y$ and $q$ in Equ.3. The Equ. 3 can be rewritten as Equ.5 around an operating point $\left(M_{T 0}, H_{0}, n_{0}, Y_{0}, Q_{0}\right)$.

$$
\begin{aligned}
& m_{t}=\frac{\partial m_{t}}{\partial h} h+\frac{\partial m_{t}}{\partial f} f+\frac{\partial m_{t}}{\partial y} y \\
& q=\frac{\partial q}{\partial h} h+\frac{\partial q}{\partial f} f+\frac{\partial q}{\partial y} y
\end{aligned}
$$

This equation can be linearized around each operating point, i.e. the six partial differences are considered as constant coefficients in a small operating zone. However it is very hard to get the six precise coefficients at each operating point. Large error may be introduced in the calculation.

Next modification is made on this model : the six partial differences are assumed to be always constant in the full operating range of turbine:

$$
\begin{aligned}
& m_{t}^{\prime}=e_{h} h+e_{f} f+e_{y} y \\
& q=e_{q h} h+e_{q f} f+e_{q y} y
\end{aligned}
$$

But the efficiency of effective energy coversion is nonlinear :

$$
m_{t}=\eta(y) m_{t}^{\prime}
$$

Because the hydraulic dynamics is much faster than the mechanic dynamics of turbine-generator group, only the static relation between the energy conversion efficiency and the wicket gate opening is considered here. 


\subsubsection{Generator and electric grid}

The dynamic property of generator and electric grid is described as next:

$$
\left(T_{a}+T_{b}\right) d f / d t+e_{g} f=m_{r}-m_{g}-m_{r}
$$

$T_{a}$ is a time constant which reflects the momentary of inertia of the turbine-generator group ; the time constant $T_{b}$ and the coefficient $e_{g}$ depend on the momentary property of the electric network, reflecting the capacity of the network. They are equal to zero when the generator is not sychronized. When the network is enormous large, $T_{b}$ is considered infinite large as the generator is synchronized, i.e. the frequency (speed of turbine-generator) is always constant. $m_{r}$ is resistant and $m_{g}$ is electric load.

The sampling period $T$ should be adaptive to the characteristic parameters of hydro-electric generating unit, especially that of hydraulic subsystem. And it must be synchronized with standard clock.

\subsection{Hardware interfaces}

Following essential hardware interfaces are required to perform the real time simulation and performance measurement of hydro-turbine governing system.

(1) Wicket gate opening measurement channel (analogue) :

(2) Wicket gate opening control signal input channel (analogue) ;

(3) Wicket gate opening output channel : this channel is used to output the simulated wicket gate opening like a wicket gate opening sensor ;

(4) Speed measurement channel ;

(5) Speed signal generation channel ;

(6) Digital signal input/output channels.

\section{III — PERFORMANCE MEASUREMENT}

The definitions of the performances of hydro-turbine governing systems are presented with reference to [1,2]. The measurement of these performances by use of HTGSMS are described.

\subsection{Static performances}

(1) Speed dead zone : the speed dead zone of a governor is defined as the maximum speed change to which the wicket gate opening is insensitive. It is denoted as $i_{x}$. The measuring unit is percentage of the rated speed. It should be smaller than $0.02 \%$.

This criteria is measured alone with the governor (if the actuator is absent, the simulation of actuator will be enabled). So the valve before the wicket gate must be closed to prevent the turbine-generator group from start-up if the test is carried out in field.

A speed signal is generated to simulate the speed of group. The speed signal is firstly set at $100 \%$. The speed set point of the governor is fixed at $100 \%$. The speed dead zone at a certain wicket gate opening $Y_{k}$ is measured with following procedure :

(a) Let $Y_{r}=Y_{k}$;

(b) A square wave oscillation with initial magnitude $\Delta f$ of $0.02 \%$ and constant period $T$ of for example 20 seconds is applied on the speed signal. The wicket gate opening is observed.
If the wicket gate opening correspondingly responses the speed signal oscillation, $i_{x}$ is smaller than or equal to $0.02 \%$. $\Delta f$ is decreased step by step (the decrement of each step, $\delta f$ should be smaller than or equal to $0.002 \%$ ) until the wicket gate opening does not have corresponding response. The exact speed dead zone is determined by next inequality :

$\Delta f<i_{x} \leq \Delta f+\delta f$

$\Delta f$ is the magnitude of the square wave oscillation to which the wicket gate opening begins to have not corresponding response.

Otherwise $i_{x}$ is larger than $0.02 \%$. The magnitude of oscillation is increased step by step (the increment of each step should be smaller than or equal to $0.002 \%$ ) until the wicket gate opening does have corresponding response. The exact speed dead zone is determined by next inequality :

$\Delta f-\delta f<i_{x} \leq \Delta f$

$\Delta f$ is the magnitude of the square wave oscillation to which the wicket gate opening begins to have corresponding response.

The speed dead zones corresponding to at least three wicket gate openings (for example $20 \%, 50 \%$ and $80 \%$ ) should be measured. Only if the speed dead zones at all the selected wicket gate openings set points are smaller than $0.02 \%, i_{x}$ of the governor is smaller than $0.02 \%$.

(2) Static regulation characteristics. In steady state, the linear relation described in Equ.9 (or Equ.10) between the speed $f$ and the power output $p$ of generator (or the wicket gate opening $y$ ) should be satisfied.

$$
\begin{aligned}
& f=-e_{p}{ }^{*} p+\left(e_{p}{ }^{*} p_{r}+f_{r}\right) \\
& f=-b_{p}{ }^{*} y+\left(b_{p} * y_{r}+f_{r}\right)
\end{aligned}
$$

Here $f_{r}, p_{r}$ and $y_{r}$ are the set points respectively of speed, power and wicket gate opening of the governor: $e_{p}\left(\right.$ or $\left.b_{p}\right)$ is the coefficient of the permanent speed droop.

This relation is the static regulation characteristics of the governor. Its measurement is usually based on Equ.10. It is carried out alone with the governor like the measurement of the speed dead zone.

$f_{r}$ is fixed at $100 \%$ and $y_{r}$ is fixed at $50 \%$. A speed signal is generated to simulate the speed of group. It is firstly set at $100 \%$. Three parameters $f_{u}, f_{l}$ and $d_{f}$ are selected according to the following principles :

- $f_{u}-100 \%=100 \%-f_{l}$;

- $y_{l-l}$ and $y_{l}$, calculated from Equ.10 according to speeds $f_{t^{-}}$ $d_{f}$ and $f_{l}$, should satisfy constraints $y_{l}>90 \%$ and $y_{l-l} \leq$ $100 \%$;

- $f_{u}-f_{l}=n * d_{f}, n$ is an integer, larger than or equal to 10 .

The speed signal is firstly changed to $f_{u}$. Then it is slowly tuned from $f_{u}$ to $f_{l}-d_{f}$ and stopped for wicket gate opening acquisition at $f_{k}=f_{u}-k * d_{f}, k=0,1, \ldots, n . n+l$ wicket gate openings $y_{u k}$ are correspondingly obtained.

Then the speed signal is slowly tuned from $f_{i}-d_{f}$ to $f_{u}$ and stopped for wicket gate opening acquisition at $f_{k}=f_{l}+(n-k) * d_{f}$, $k=n, \ldots, l, 0 . n+l$ wicket gate openings $y_{d k}$ are correspondingly obtained.

From these acquisitions, two linear lines described by the following equation can be obtained by the least square method.

$$
\begin{array}{ll}
f=-a_{l u^{*}}{ }^{2} y+a_{0 u} & \text { in open direction } \\
f=-a_{l d}{ }^{*} y+a_{O d} & \text { in close direction }
\end{array}
$$


Here

$a_{1 s}=\frac{\sum_{k=0}^{n} y_{s k} \sum_{k=0}^{n} f_{k}-(n+1) \sum_{k=0}^{n} f_{k} * y_{s k}}{(n+1) \sum_{k=0}^{n} y_{s k}{ }^{2}-\left(\sum_{k=0}^{n} y_{s k}\right)^{2}} \quad s=u$ and $d$

$a_{0 s}=\frac{\sum_{k=0}^{n} f_{k}-a_{1 s} \sum_{k=0}^{n} y_{s k}}{n+1} \quad s=u$ and $d$

These two lines are the measured static regulation characteristics of the governor, in respectively open and close directions of the wicket gate. $a_{l s}$ is the measured permanent speed drop. The difference between two lines reflects the dead zone of the regulation of the governor. The variant between the linear line and the measured points reflects the non-linear degree of the static regulation characteristics.

\subsection{Dynamic performances}

\subsubsection{Start-up operation}

Although there is no quantity criteria for start-up operation, following requirements must be satisfied :

- The group should pass the low speed $(0 \sim 60 \%)$ as fast as possible ;

- No overshoot of speed is expected;

- The regulation time should be minimized so that the generator can be synchronized with the electric grid as soon as possible.

The start-up operation algorithm may be carefully optimized with HTGSMS.

\subsubsection{Speed fluctuation in unsynchronized operation condition}

The speed fluctuation is measured when the speed set point is fixed at $100 \%$ and the generator is unsynchronized with electric grid, after start-up operation. The time window of measurement $T$ depends on the frequency of speed fluctuation. At least three cycles of speed fluctuation should be covered in the selected window.

The speed fluctuation should be inferior to $\pm 0.15 \%$ of the rated speed.

3.2.3. Response to step changes on speed set point in unsynchronized operation condition.

The responses of speed and wicket gate opening to step changes on speed set point are recorded and the overshoot $M_{p}$ and the regulation time $T_{s}$ of speed response are measured.

$$
M_{p}=\frac{F_{p}-F_{s}}{F_{s}-F_{c}} \times 100 \% M_{p} \geq 0
$$

$F_{c}$ is the Current speed set point before step change ; $F_{g}$ is the Goal speed set point of the step change ; $F_{p}$ is the maximum (for step-up change) or minimum (for step down change) speed after the step change; $F_{s}$ is the steady speed after the step change, $F_{s} \cong F_{g}$.

$T_{s}$ is defined as the time duration from the instant at which the step change is applied to the instant from which the speed permanently entered into a band $\left[F_{s}-\delta f / 2, F_{s}+\right.$ $\delta f / 2]$.
A control parameter optimization algorithm is designed and integrated into HTGSMS. This algorithm synthesizes the responses to a set of special arranged step changes on speed set point and tunes the control parameters in order to optimize the control performance [4].

The dynamic performances in unsynchronized operation condition is essential to the stability of hydro-turbine governing system.

\subsubsection{Dead time of regulation $T_{q}$}

$T_{q}$ is defined as the time delay of the wicket gate opening response to a certain speed change.

According to the definition, $T_{q}$ can be measured in following way : a step up change of $0.05 \%$ is applied on the speed signal and the wicket gate opening is observed. $T_{q}$ is equal to the time delay from the instant of the step change to the instant at which the wicket gate opening starts to steadily decrease.

Another method to measure $T_{q}$ is to use the speed increase after 10 25\% load rejection as input signal. Then $T_{q}$ equals to the time delay from the instant at which the power output of generator is completely cut off to the instant at which the wicket gate opening starts to steadily decrease. $T_{q}$ should be smaller than or equal to 0.2 second.

\subsubsection{Dynamics after 100\% load rejection}

There are four performance measurements : maximum speed raise $f_{m}$, maximum pressure raise $p_{m}$, speed regulation time $T_{p}$ and speed oscillation cycles $N / 2$.

$T_{p}$ is defined as the time period from the instant at which the wicket gate opening firstly increases after $100 \%$ load rejection to the instant from which the magnitude of speed oscillation is inferior to $\pm 0.5 \%$ of rated speed. It should be less than 30 seconds.

$N$ is defined as the number of the half cycles of the speed oscillation with magnitude superior to $0.5 \%$ of rated speed. It should be less than or equal to 2 .

The maximum speed raise must be under the limit of runoff speed. The pressure raise must be inferior to the maximum safety pressure of hydraulic subsystem. These two limits are very important to the safety of hydro-electric generating unit.

\subsubsection{Control parameters measurement of PID-type controller}

The PID coefficients $K_{p}, K_{i}$ and $K_{d}$ can be measured according to the wicket gate control output of the controller and corresponding speed signal input.

The measured parameters are compared with the preset ones to verify the implementation of control algorithm. The set range of each parameter can also be verified.

\section{D TEST RESULTS}

Both static and dynamic performances can be automatically measured by HTGSMS with three possible configurations :

- the function and control performance of a controller can be tested and measured with simulated actuator and generating unit ;

- the function and control performance of a governor can be tested and measured with simulated generating unit ;

- the function and control performance of a complete hydro turbine governing system can be tested and measured in field. 


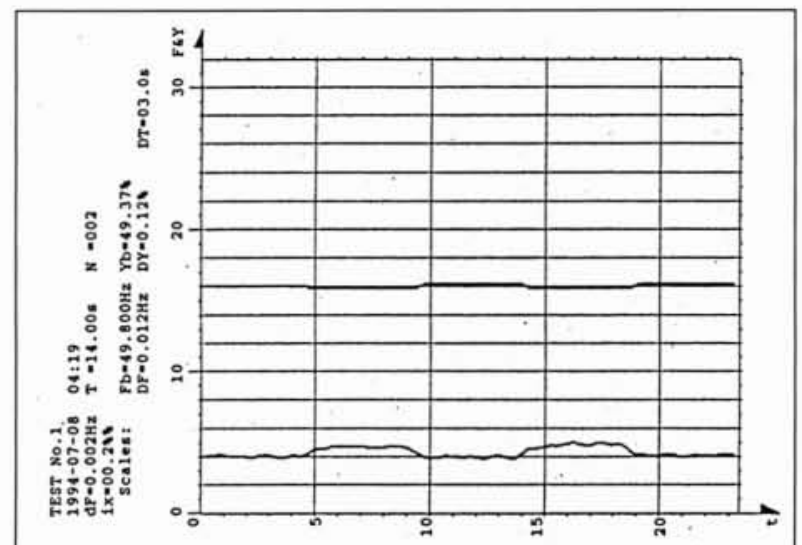

a)

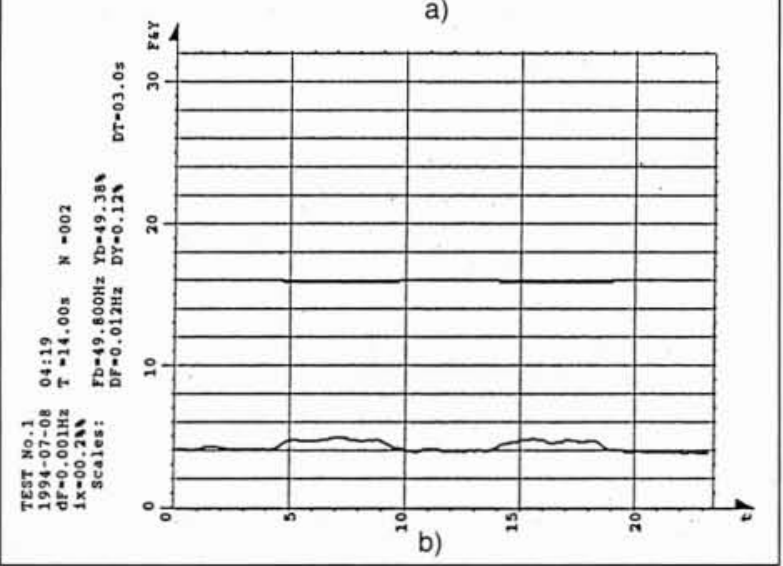

5. Measurement of Speed Dead Zone*

HTGSMS has been widely applied to the tests of controllers as well as governors in research departments, manufacturing workshops and hydro power plants in China. The field test results in hydro power plants were presented in $[3,4]$. Here gives some test results of controller with the real time simulation of both actuator and generating unit.

(1) Speed dead zone of regulation : the speed is represented by the frequency of the A.C. electric output of generator.

The frequency corresponding to rated speed is $50 \mathrm{~Hz}$.

Fig.5 shows the last two steps of the measurement of the speed dead zone. In Fig.5a, the magnitude $d F$ and period $T$ of square wave oscillation are respectively $0.004 \%$ $(0.002 \mathrm{~Hz})$, and 14 seconds. The wicket gate opening correctly responses the change on the speed signal. In Fig.5b, $d F$ is automatically tuned to $0.002 \%(0.001 \mathrm{~Hz})$. Although the wicket gate opening has correct response to the change on speed signal, the test terminates with the result $i_{x} \leq 0.002 \%$.

(2) Static regulation characteristics. Three parameters $f_{u}, f_{l}$ and $d f$ are respectively $F l=51.200 \mathrm{~Hz}, F D=48.80 \mathrm{~Hz}$ and $d F=0.200 \mathrm{~Hz}$. The $n(=13)$ speed (frequency) points and the corresponding measured wicket gate openings in both open and close directions are listed in Tab.2.
The two lines of the measured static regulation characteristics in respectively open and close directions of the wicket gate are demonstrated in Fig.6.

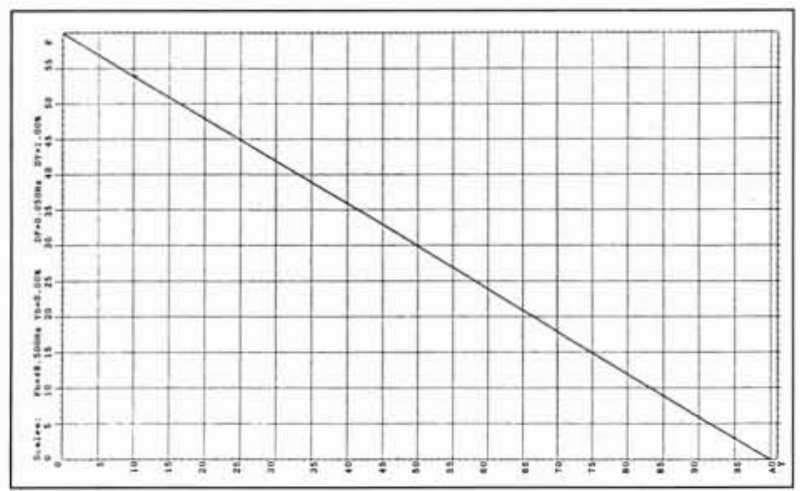

6. Verification of Static Regulation Characteristics.

Scale: horizontal axis-wicket gate opening Y DY/division on base of $Y b$. vertical axis-frequency (speed) FDF/division on base of $F b$;

The measured permanent speed drop $b p$ is equal to $6 \%$. The non-linear degrees in open and close directions are respectively $0.054 \%$ and $0.052 \%$. The dead zone of regulation is $0.01 \%$.

(3) Start-up operation : the start-up operation of "turbinegenerator group" is recorded in Fig.7. It can be seen that the wicket gate was firstly opened to a start-up opening which is much larger than no-load opening and the speed of turbine-generator raised very quickly. When the speed reached about $90 \%$ of the rated speed, the wicket gate was closed down to near no-load opening and switched to automatic regulation. The speed smoothly increased to the rated value.

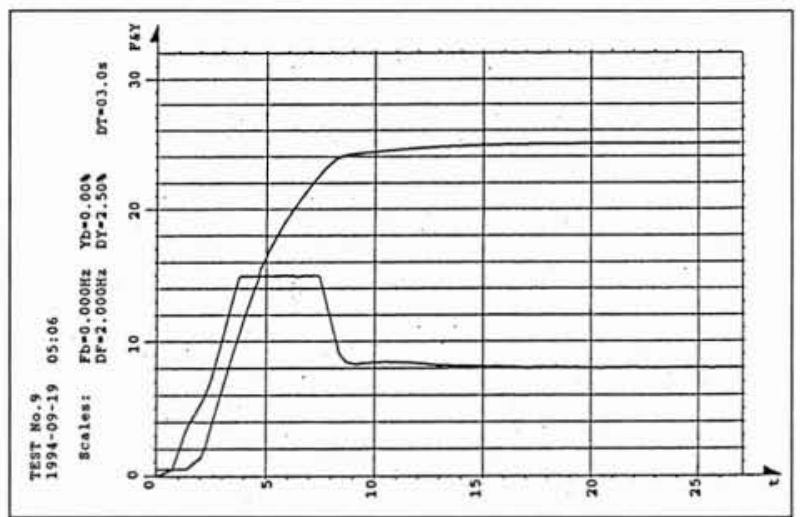

7. Record of Start-up Operation*

(4) Speed fluctuation : the time window $T$ is equal to 3 minutes. The speed fluctuation is obtained by following equation: $\delta f=(F h-F l) \times 2 \%=(50.024-50.004) \times 2 \%=$ $0.04 \%$

\begin{tabular}{|c|c|c|c|c|c|c|c|c|c|c|c|c|c|}
\hline$f(\mathrm{~Hz})$ & 51.20 & 51.00 & 50.80 & 50.60 & 50.40 & 50.20 & 50.00 & 49.80 & 49.60 & 49.40 & 49.20 & 49.00 & 48.80 \\
\hline$y-$ open (\%) & 10.25 & 16.75 & 23.35 & 29.98 & 36.57 & 43.18 & 49.78 & 56.32 & 62.96 & 69.59 & 76.18 & 82.77 & 89.36 \\
\hline$y-\operatorname{close}(\%)$ & 10.37 & 16.92 & 23.56 & 30.14 & 36.81 & 43.39 & 49.92 & 56.51 & 63.11 & 69.76 & 76.34 & 82.94 & 89.50 \\
\hline
\end{tabular}




\section{Applications industrielles de la mécanique des fluides}

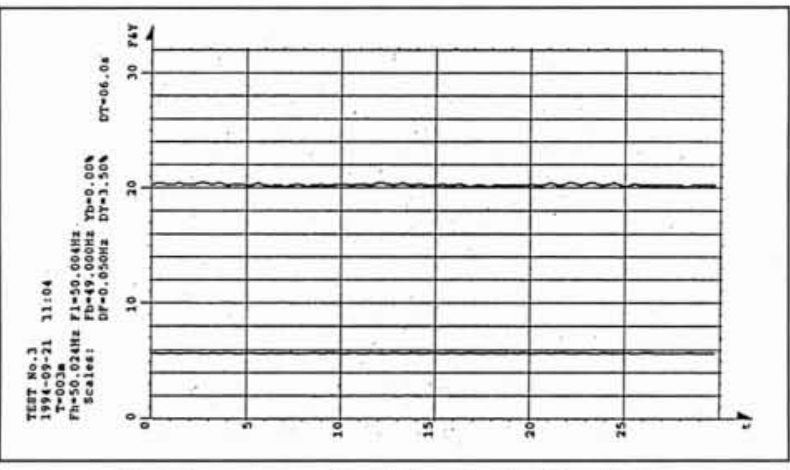

8. Measurement of Speed Fluctuation*

(5) Step change of speed set point : the speed set points before and after step change are respectively $F c=49.000 \mathrm{~Hz}(98 \%)$ and $F g=51.000 \mathrm{~Hz}(102 \%)$; the overshoot $M p$ and the settling time $T s$ are respectively equal to $13.8 \%$ and 15.0 seconds in Fig.9.

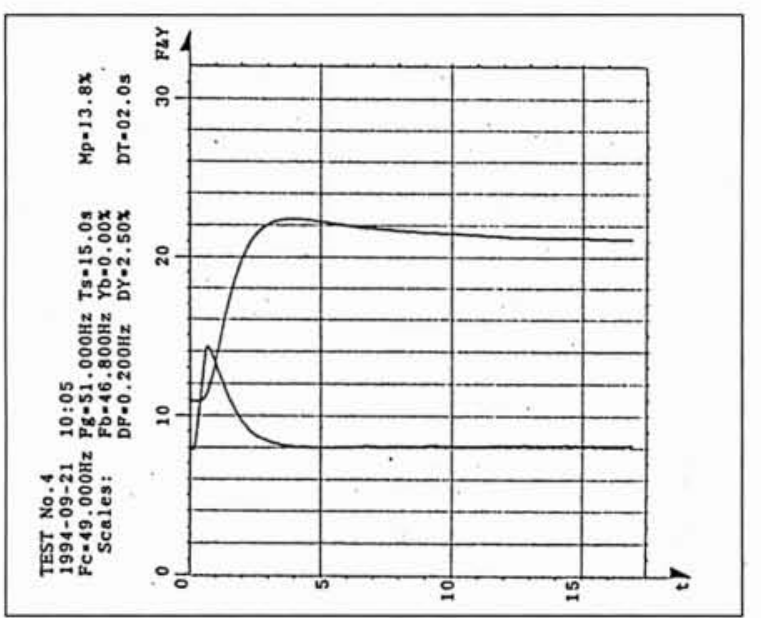

9. Response to Step Change on Speed Set Point*

(6) Control performance optimization : one of a set of special arranged step changes on the speed set point for performance optimization is shown in Fig.10.

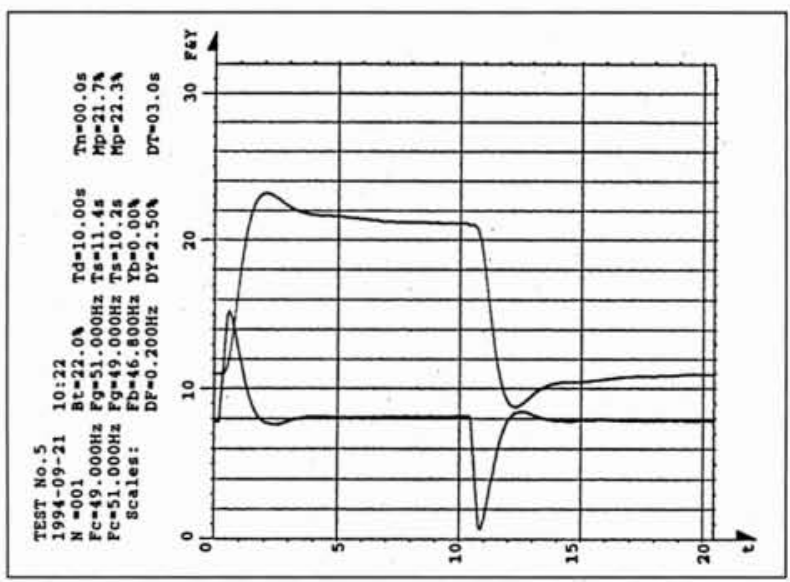

10. One of Special Arranged Step Changes for Performance Optimization*

After all the arranged step changes have been completed, the responses would be synthesized and the control parameters would be tuned to achieve better performance [4].
(7) $100 \%$ load rejection : the load rejection occured at the instant $\mathrm{t}=0$. The wicket gate is firstly quickly closed to a certain opening, e.g. no-load opening, to avoid too important speed raise of turbine-generator. Then the close speed is slowed down to reduce harmful pressure raise. The maximum speed raise $f_{m}$ reaches $137.9 \%$; the regulation time $T_{s}$ is 8.4 seconds; and $N=1$.

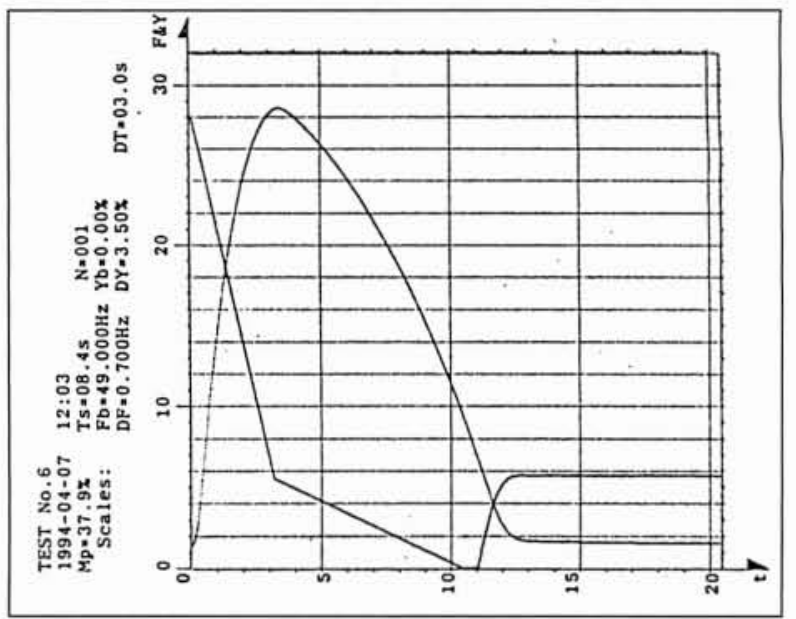

11. $100 \%$ Load Rejection*

(8) 10 25\% load rejection : the load rejection also occured at the instant $\mathrm{t}=0$ in Fig.11. The dead time $T_{q}$ was measured to be 0.13 second.

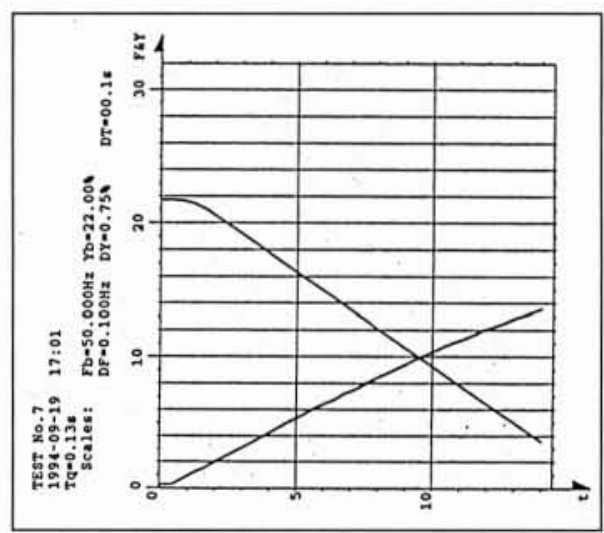

12. $10 \sim 25 \%$

Load

Rejec-

tion*

(9) Control parameter measurement : the PID parameters of the controller were set at $K_{p}=4.2, K_{i^{\prime}}=0.4, K_{d}=2.0$. The measured ones are $K_{p}=4.1, K_{i}=0.4, K_{d}=1.9$.

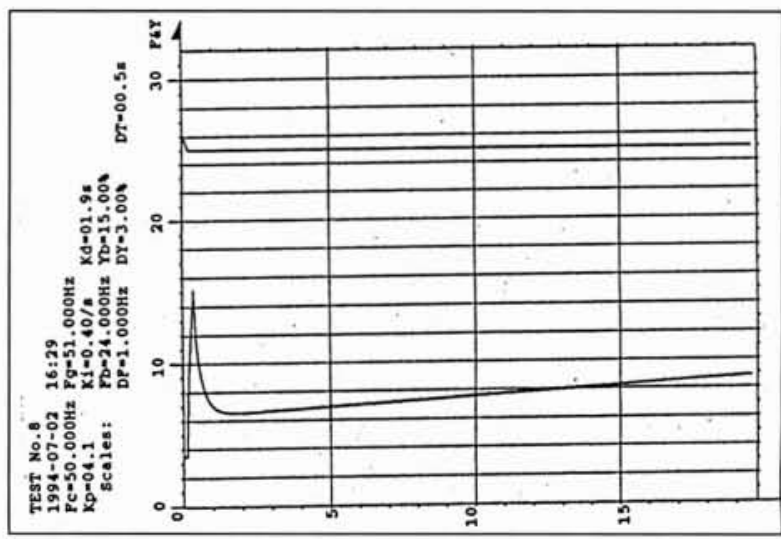

13. Measurement of PID Parameters*

*Scales: horizontal axis - time t DT/division; : vertical axis - frequency (speed) $\mathrm{F}$; DF/division on base of $\mathrm{Fb}$; — wicket gate opening $\mathrm{Y}: \mathrm{DY} /$ division on base of $\mathrm{Yb}$. 
All of these results are in accordance with the field test results in hydro-power plants.

\section{FURTHER DEVELOPMENTS}

Further developments on the real time simulation techniques of hydro power plants and their applications include following two aspects.

\subsection{Techniques}

\subsubsection{Modeling techniques}

The models of a hydro power plant are categorized to analogue models and logic models.

- Analogue models describe the behaviors and the properties of all the components with continuous states, such as hydraulic subsystem, turbine-generator groups and their controllers, electric network (from the point of view of the interaction between hydro power plant and network), etc.

- Logic models mainly represent the sequential operations and the protections of the hydro power plant.

All models should be highly modularized so that different components can be separately modeled by their own experts and the replacement of a component in a "hydro power plant" has no influence on the models of other components. Furthermore both analogue and logic models must be easily programmed on computers.

To meet these requirements, the graphic modeling approaches could be adopted, for example, bond graph models for the analogue part and Petri net models for logic part.

\subsubsection{Programming techniques}

A data base including a component library will be constructed. It is initially set with the information of a particular application. And it will be enriched in the late use, with an updating tool. For example, new components, new specification(s) or new model(s) of a component can be added to the data base by use of the updating tool.

The user can take the components in the library (such as pipe line, surge tank, turbine, governor, generator, voltage regulator etc.) to graphically construct a "hydro power plant" on the computer.

A software compiler and linker will be built to translate the graphic "hydro power plant" into an executable file for real time simulation of the hydro power plant. This executable file can run under the supervision of a software monitor. This monitor allows the user to examine the state(s) of the "hydro power plant" during simulation.

The output(s) of any component in the "hydro power plant" can be obtained via hardware interfaces of computer. And the input(s) of any component in the "hydro power plant" can be fed in, also via the hardware interfaces of computer. In this way, a real component can replace the simulated one and participate the operation of the simulator. Some of these techniques are already available.

\subsection{Applications}

A simulator of the hydro power plant will be built up on base of the above techniques. This simulator could be widely used :

- to educate future engineers for hydro electric engineering and train operators for hydro power plants ;
- to test new sequential operation program(s), new control algorithm(s), and the equipment (or subsystems) such as governors and voltage regulators, at different stages of research, design, manufacture, installation, maintenance, and renovation ;

- to detect and diagnose the faults in real plant by running the simulator in parallel with the real plant. This will also be another application example of analytical redundancy techniques such as model-based fault detection techniques [5] ;

- and even to design a new hydro power plant.

These applications will have great significance in education, research, design, manufacture and operation of hydro power plants and their equipment.

\section{VI — CONCLUSIONS}

The real time simulation and performance measurement techniques for hydro turbine governing systems are very welcomed by both research and industrial departments in China. Comprehensive tests with the real time simulation facilities before the service on real hydro generating units not only largely reduced the time required for development and installation but also enhanced the performance and reliability of hydro turbine governors.

The successful applications persuasively demonstrated the significance of simulation, especially real time simulation, and automatic performance measurement in research, design, manufacture, and operation.

The project on the real time simulation techniques of overall hydro power plant is feasible with today's development on computer science and engineering. It will have bright application future.

\section{REFERENCES}

[1] Technical conditions for hydro turbine governing systems and oil pressure devices, GB9652-88, Technical Standard of People's Republic of China, 1989.

[2] General regulations on the adjustment and the test of the electrichydraulic regulation system and equipment of hydro turbines, DL496-92, Technical Standard of Electric Industry, People's Republic of China, 1989.

[3] LI ZHAOHUI, Field tests with a prototype microcomputer-based hydro turbine governors, Large Electric Machine and Hydraulic Turbine, Sum.125, No.2, 1996.

[4] LI ZHAOHUI and O.P. MALIK, An Orthogonal Test Approach Based Control Parameter Optimization and Its Application to Hydro Turbine Governor, submitted to IEEE 1997 Summer Meetings.

[5] LI ZHAOHUI et al., Model Based Fault Detection for Hydro Turbine Governing Systems, Proc.of CESA'96, IMACS \& IEEE Multiconference, Symposium on Control, Optimization and Supervision, Vol.1 of 2. France-Lille, July 9 12, 1996. 